by Borg (see, e.g., Borg 1978; also Borg et al. 1987). Invariant relations have been found between heart rate, blood lactate and ratings $(\mathrm{R})$ of perceived $(\mathrm{P})$ exertion $(\mathrm{E})$ according to the "RPEscale" (probably one of the most commonly used psychophysical methods, with several hundred studies performed in the last few years and approximately one million people exposed to it every year), and the "CR-scale" (category scale with ratio properties, Borg 1982). For interpersonal comparisons a "rating scale" may be superior to a "ratio scale"!

Guilford (1936) provides a good review of rating scales (with an example from descriptions of weather in 1805 reported by Titchener). He did not make the same mistake as Stevens and most psychophysicists, including Fechner - notable exceptions are Parducci (1984), Heller and the Würzburg group (see, e.g., Heller 1990), Borg (1962), and a few others. Guilford understood the importance of "descriptive terms" and "landmarks." He concludes that the rating methods "have overshadowed the applications of all the other evaluative methods based upon personal judgements" (p. 263). But he also points out how "indispensable" these methods are to theoretical psychology. I definitely agree with him, but would also like to stress the importance of using the best properties of the ratio scaling methods for general, relative intensity evaluations, together with the best properties of the category-rating methods for level determinations and interindividual comparisons. This use of "meaningless" numbers with "meaningful" linguistic terms will improve the possibilities of understanding human communication and the problems of intersubjectivity.

\section{The phantom limb extrapolation}

\section{Willard L. Brigner \\ Psychology Department, Appalachian State University, Boone, NC 28608 Electronic mall: brignerwl@conrad.appstate.edu}

In tracing the development of Fechner's inner psychophysics, Murray seems apologetic (vid. sect. 1.2, para. 5). In presenting signal detection theory as the major development of Fechnerian inner psychophysics, he notes, no doubt correctly, that there are only "commonalities of interest" between signal detection theory and Fechnerian inner psychophysics. In other words, the Fechnerian tradition should be considered incidental in the development of signal detection theory and should not be considered conative. This approach to the topic of inner psychophysics seems feckless. Instead, when Murray alludes to the neurelectric aspects of a stimulus (e.g., sect. 1.2, para. 2) or to neurelectric measures of receptor response (e.g., Note 3 and sect. 1.3.4, para. 3), one would hope that the problem at "the heart of inner psychophysics" (sect. 1.2, para. 2) is to be addressed, but it never is.

In discussing inner psychophysics, Murray could have cited some long-standing electrophysiological data which support Fechner's Law in that the frequency of neural firing has been shown to be in approximately direct proportion to the log of the stimulus intensity. This relationship has been demonstrated, for example, by recording from the nerve fiber of an isolated ommatidium in the Limulus eye (Ratliff \& Hartline 1959). It has also been demonstrated by recording from single axons of stretch receptors of the muscle spindle in the cat (Patton 1960). Consistent with the power law, in recordings from single nerve fibers subserving mechanoreceptors in the skin of the monkey, the $\log$ rate of neural firing has been shown to be approximately directly proportional to the log stimulus pressure (Werner \& Mountcastle 1965). (Note that the question of whether the appropriate law is logarithmic or a power law extends to electrophysiological data.)

Of course, the electrophysiological data and the sensation scaling data offer an interesting implication. The implication is that frequency of neural firing (at the receptor level) and sensation strength (or psychological magnitude) are interchangeable, and an interchangeability of something physiological with something psychical suggests inchoate equivalences regarding, as Murray describes it, "the heart of inner psychophysics ... the 'mind/brain' problem ..." (sect. 1.2, para. 2). Of course, Murray's reticence in developing inner psychophysics along these lines can be understood, since one rarely encounters the Cartesian issues of mind-body dualism, innate ideas, and so on, in the current psychological literature. But there are exceptions - such as the following.

Based upon work with patients experiencing phantom limbs, phantom vision, and phantom hearing, Melzack (1992) extrapolates the experience of phantom limbs to the experience of a phantom body. He writes that "The brain does more than detect and analyze inputs; it generates perceptual experience . . . [in fact] the brain generates the experience of the body.... Sensory inputs merely modulate that experience...." (p. 126). Although "the experience of the body" may not be on Descartes' list of innate ideas, Melzack would seem to be offering it as a candidate. His brain-generated experience of the body provides an amusing contrast to Locke's discussion of the tabula rasa, where there was a body and the phantom was phenomenal experience. So Murray's development of inner psychophysics would have been more effective if it had possessed some of the temerity of the phantom limb extrapolation. ${ }^{1}$

\section{NOTE}

1. A final trivial point: Murray fails to distinguish between border contrast and Mach bands (vid. sect. 2.2, para. 2). The increment/decrement in apparent brightness at borders, where there is an abrupt step in intensity, is usually referred to as border contrast. If the intensity distribution includes an intensity gradient linking different levels of intensity, the increments/decrements in apparent brightness at the gradient end(s) are referred to as Mach bands.

\section{From metaphysics to psychophysics and statistics}

\section{Gerd Gigerenzer}

Department of Psychology, University of Chicago, Chicago, IL 60637 Electronic mail: giger@midway.uchicago.edu

David Murray has reminded us that the issues raised by Gustav Theodor Fechner are still the stock-in-trade of modern psychophysical laboratories. I will follow Murray's lead here and ask the question whether (and how) Fechner's issues and their modern offspring are related to Fechner's philosophy.

Fechner's metaphysics is a unique blend of monism, pantheism, panpsychism, and indeterminism, for which no single English word exists. Three books published before the Elemente der Psychophysik (1860), none of which has been translated into English, contain the elements of Fechner's metaphysics. In Das Büchlein vom Leben nach dem Tode (first published in 1836 under the pseudonym Dr. Mises), Fechner argued that man lives not once, but thrice. The first life is a state of constant sleep, the second is a periodic alternation between sleeping and waking, the third is eternal waking. The transition is through birth and death. To be awake means to be conscious, and consciousness means that the "so-called psychophysical activity rises above that degree of strength which is called threshold" (p. 51). In Nanna (1848) Fechner argued that plants have souls. In Zend-Avesta (1851) he explained why the earth and planets have souls as well.

The idea that not just we humans have consciousness flows from his monism: Mind and body are the same thing, they just look different as seen from inside and outside, respectively. If you look into yourself, you perceive psychical processes; if I look at you, I perceive a material entity. The essential unity of mind 
and body implies that not only humans are conscious, but plants and planets as well. Fechner points to Kepler's similar ideas in Harmonia mundi, where the earth is a living creature who alternates between sleeping and waking states. Finally, nature seen as a whole has a soul as well, which is God. Seen from the perspective of Fechner's monism, to search for causal laws that link the psychical to the material or vice versa, is a waste of time. There are no such laws between mind and body: There is identity, no succession. Causal laws only hold within each of the two realms. This brief sketch of Fechner's metaphysics must suffice for present purposes.

Did aspects of Fechner's scientific work originate in his metaphysics? If so, does psychophysics still bear these birthmarks? I will attempt a partial answer by examining Fechner's two major treatises on psychophysics and statistics.

1. In his Elemente der Psychophysik (1860), Fechner explicitly links his psychophysical program to his metaphysical work (e.g., vol. 2, p. 543). Several writers, from Boring (1929, p. 269) to Murray (sect. 1.1) have pointed out that Zend-Avesta already contained Fechner's program of psychophysics. More specifically, the common interpretation seems to be (a) that Fechner's program of measuring sensation and determining psychophysical functions was a means to prove monism, that is, to demonstrate the essential unity of mind and body through a simple mathematical equation, and (b) that in Zend-Avesta, Fechner intuited the logarithmic shape of the psychophysical function before he did any experiments. I have two comments.

(a) Psychologists may not have embraced Fechner's program in order to grasp immortality (Fechner's own aim), but they did nonetheless adopt the program as a means to their own ends. Does this mean that its scientifically embarrassing origins are accidental to the history of psychophysics, amusing at best? I don't think so. Fechner's metaphysics may well have given the history of psychophysics an impulse in a particular direction. Here is the argument. There are many possible programs of psychophysics. Not every program can serve as a means to prove monism. Programs geared toward identifying psychophysical functions, whether Fechner's or Stevens's, can serve that end. Programs that reject a one-to-one relationship between sensation and stimulus, however, would not have served that purpose - from Gestalt psychology to Brunswik's (1934) multidimensional psychophysics to the psychology of contextual effects (e.g., Parducci 1982). That is, if one assumes a parallelism between brain states and perception, but complex Gestalt laws rather than a parallelism between these two and the physical world, then no simple psychophysical function exists. Or, if contextual effects result from coherence systems and perceptual compromises, as in Brunswik's multidimensional psychophysics, again no single psychophysical function exists (Gigerenzer \& Murray 1987, Chapter 3). It is, of course, not easy to say what could count as evidence for the suggested impulse from metaphysics, and the pointed neglect of contextual effects in psychophysics was no doubt overdetermined. The best $I$ can do here is to point to the difficult time contextual effects have had throughout the history of psychophysics, often ignored or downplayed as mere "nuisances" or "response biases" (on this see Birnbaum 1989). The questions that define the history of psychophysics seem to exclude contextual effects in favor of psychophysical functions.

(b) My second comment also addresses the context of discovery: Where does the logarithmic shape of the psychophysical function come from? The idea is already in the Zend-Avesta, although it does not seem necessary for proving monism; a power law would have done just as well. Murray (1987, p. 77) has pointed to potential extrametaphysical origins: The logarithmic laws formulated by Daniel Bernoulli or by astronomers may have served Fechner as an analogy. There exists one further possible origin, which is more closely linked to Fechner's own research. Fechner had spent two years of research
(1829-1831) on the galvanic battery, which earned him a chair of physics at the University of Leipzig. Fechner's research was based on Ohm's path-breaking treatise on that subject. As Stigler (1986, p. 243) reminds us, Ohm's first paper of 1825 postulated the formula

$$
V=m \log (1+x)
$$

for the relationship between the loss of force of current $V$ and the length of wire $x$. Fechner proposed the same logarithmic relationship for the psychophysical function,

$$
S=k \log R,
$$

where $S$ is the sensation, $R$ the physical stimulus, and $k$ a constant value. Thus, the idea of a logarithmic psychophysical function may well be by analogy to Ohm's law. To summarize the gist of these two comments: In the history of psychophysics, Fechner's metaphysics may well have played a role. Metaphysics has helped to select one class of programs and to exclude others. Within that selected class, however, an analogy from the physical sciences seems to be the source of a particular expression.

2. In his Kollektivmasslehre (posthumously published in 1897), Fechner is silent about any connections with his metaphysics, except for a hint in the preface (p. vi). In the last decades of his life, Fechner became reluctant to speak about metaphysics when addressing a scientific audience, realizing how unpopular his metaphysical mission was among his fellow scientists. The Kollektivmasslehre is a theory of frequency distributions, and its goal is the description of kollectivs (such as the height of Belgian soldiers or the dimensions of religious paintings). Its tools are asymmetric generalizations of Gaussian distributions, measures of central tendencies, variability and correlation, and tests of statistical independence. In a seminal article, Heidelberger (1987) has traced its relation to Fechner's philosophical indeterminism. In Fechner's monism, the mental is not causally determined by the material, rather, the mental is indeterminate in several ways, most importantly in the sense of intrinsic novelty. Novel elements emerge in the mind, leading to novel consequences. Because mind and matter are just two appearances of the same entity, true indeterminism - not just Laplacean ignorance of conditions - also holds in the physical world. Indeterminism, however, does not preclude the scientific study of nature (a remarkable view to be voiced long before chance became an indispensable part of physics). The Kollektivmasslehre was Fechner's program for studying indeterminate nature.

Fechner's program is a strictly frequentist theory of probability, in marked contrast to the classical interpretation of probability, dominant circa 1660-1840 (Daston 1988). Richard von Mises, founder of the frequentist theory in our century, is explicit about the influence Fechner had on him: "his views have served, at least for me, as a stimulus in developing the new concept of probability" (von Mises 1928/1957, p. 83). The rest of the story is well known. Frequency statistics eventually conquered psychophysics and all of the experimental social sciences, albeit not in the form of von Mises's large sample statistics, but through R. A. Fisher's (1935) small sample statistics. By this strange route, metaphysics contributed to the hardheaded empiricism of logical positivism.

To summarize: Elements of Fechner's scientific work, both in the Elemente and the Kollektivmasslehre, can be traced back to Fechner's metaphysics. This source opened new programs, but may also have inhibited the growth of others. Looking at the context of discovery, even one as mystical to our eyes as the present one, can help us understand part of the present as well as the past of psychophysics, including the origins of the kinds of question and tools that are still with us. 